\title{
Spinal CSF Leaks: Mimicker of Primary Headache Disorder in a Child
}

\author{
Sheri Harder, Robert W. Griebel, Edmond G. Lemire, Stefan Kriegler, Josh Gitlin, Shashi S. Seshia
}

Can J Neurol Sci. 2012; 39: 388-392

Spontaneous intracranial hypotension $(\mathrm{SIH})$ due to spinal cerebrospinal fluid (CSF) leaks is becoming increasingly recognized as a cause of headache in adults. ${ }^{1}$ A combination of minor trauma and developmentally 'weak' meninges has been considered to be responsible for some cases. ${ }^{1,2}$ There is also an association with autosomal dominant polycystic kidney disease, neurofibromatosis type 1, Marfan and the Ehlers-Danlos syndromes..$^{1-3}$

The onset of symptoms is typically in the fourth or fifth decade of life, females being more affected than males. Childhood presentation in the absence of Marfan syndrome is rare, ${ }^{4}$ and the diagnosis can be challenging, as illustrated by our case report.

\section{CASe Report}

A nine year old girl presented to the Pediatric headache clinic with a three month history of chronic daily headache $(\mathrm{CDH})$. She had had recurrent headaches from the age of four years. Recent headache characteristics fulfilled the International Headache Society's criteria (ICHD-II) for chronic migraine, 5,6 with features of both migraine without aura and tension-type. There were no clinical features, especially postural change or cough headache, to suggest low or high intracranial pressure (ICP). Headaches were not worsened during sports, dancing, biking, roller blading or gymnastics, and did not compromise participation in these activities or school attendance. There was no history of indirect (example, rear ended in a car) or direct trauma to her back or limbs, and she had not had lumbar punctures (LPs). The neurological and systemic examinations were normal. There were no features of a neurocutaneous disorder.

Prospective charting over the next two weeks confirmed that headaches were usually mild or moderate, rarely severe. Location was primarily occipital, but sometimes generalized. On some days they were throbbing with mild photophobia, phonophobia and nausea. On other days, headaches were dull and constant without photophobia, phonophobia or nausea. There was no vomiting at any time, and headaches were not worsened with physical activity or influenced by posture. Headaches occurred almost daily, lasted for several hours at a time, and did not wake her up from sleep. A secondary headache disorder, perhaps related to a spinal arachnoid cyst, was considered because of the occipital location of her headaches and a history of low back pain during and in between headache. The backache was not related to physical activity or posture.

$\mathrm{X}$-rays of the cervical, thoracic and lumbo-sacral spine were normal. Computed tomography (CT) of the brain, within three weeks of the initial visit, was interpreted as showing findings of raised ICP. Magnetic resonance imaging (MRI) of the head and cervical spine with and without contrast, done a week later, was also considered to be suggestive of raised ICP because of fullness of the brain; the likelihood of intracranial hypotension was also raised (by author SK) because of possible mild diffuse dural enhancement. However at a case conference, others were less certain about the findings. The MRI of the entire spine with and without intravenous gadolinium, specifically looking for evidence of arachnoid cysts and CSF leaks was normal. Neurosurgical opinion (RWG) was sought. LP under local anesthesia, in the lateral decubitus position, showed an opening pressure of $85 \mathrm{~mm}$ of water (Normal range: $100 \mathrm{~mm}-180 \mathrm{~mm}$ ) at the first needle insertion; there were 29 white blood cells (Normal 0-5 x 106/L) mainly lymphocytes, and 20 red blood cells (Normal $0 \times 10^{6} / \mathrm{L}$ ) in the CSF; the protein and glucose values were $0.57 \mathrm{~g} / \mathrm{L}$ and $3.6 \mathrm{mmol} / \mathrm{L}$ respectively (normal ranges, 0.12 to $0.6 \mathrm{~g} / \mathrm{L}$ and 2.8 to $4.5 \mathrm{mmol} / \mathrm{L}$ respectively). Smear and culture were negative. As the CSF pressure was near normal and neuroradiological findings considered ambiguous, factoring in headache description and normal examination, a decision was made to follow her up rather than proceed immediately to further, necessarily invasive, testing.

Chronic daily headache persisted over the next year, severity and characteristics being unchanged. There was no response to amitryptiline. The MRI was repeated. The study showed findings diagnostic for SIH, including downward displacement of the cerebellar tonsils, flattening of the ventral brainstem, effacement of the basal cisterns, and bowing of the infundibulum. The opening pressure, at the first LP attempt (RWG) performed under local anesthesia in the lateral decubitus position, was zero. A lumbosacral leak was demonstrated with radionuclide 99mTC DTPA cisternography. Conventional myelography (Figure 1) localized the leak to around the right S2 nerve root sleeve. Blood patching on two occasions did not

From the Department of Radiology (SH), Loma Linda Medical Center, Loma Linda, California, USA; Department of Surgery (Division of Neurosurgery) (RWG), Department of Pediatrics (Division of Pediatric Neurology) (SSS), Department of Pediatrics (Division of Medical Genetics) (EGL), Department of Radiology (SK, JG), Royal University Hospital and University of Saskatchewan, Saskatoon, Canada. Received August 22, 2011. Final Revisions Submitted November 28, 2011. Correspondence to: Shashi S. Seshia, Department of Pediatrics (Division of Pediatric Neurology), University of Saskatchewan, 103, Hospital Drive, Saskatoon, Saskatchewan, S7N 0W8, Canada. Email: sseshia@yahoo.ca 


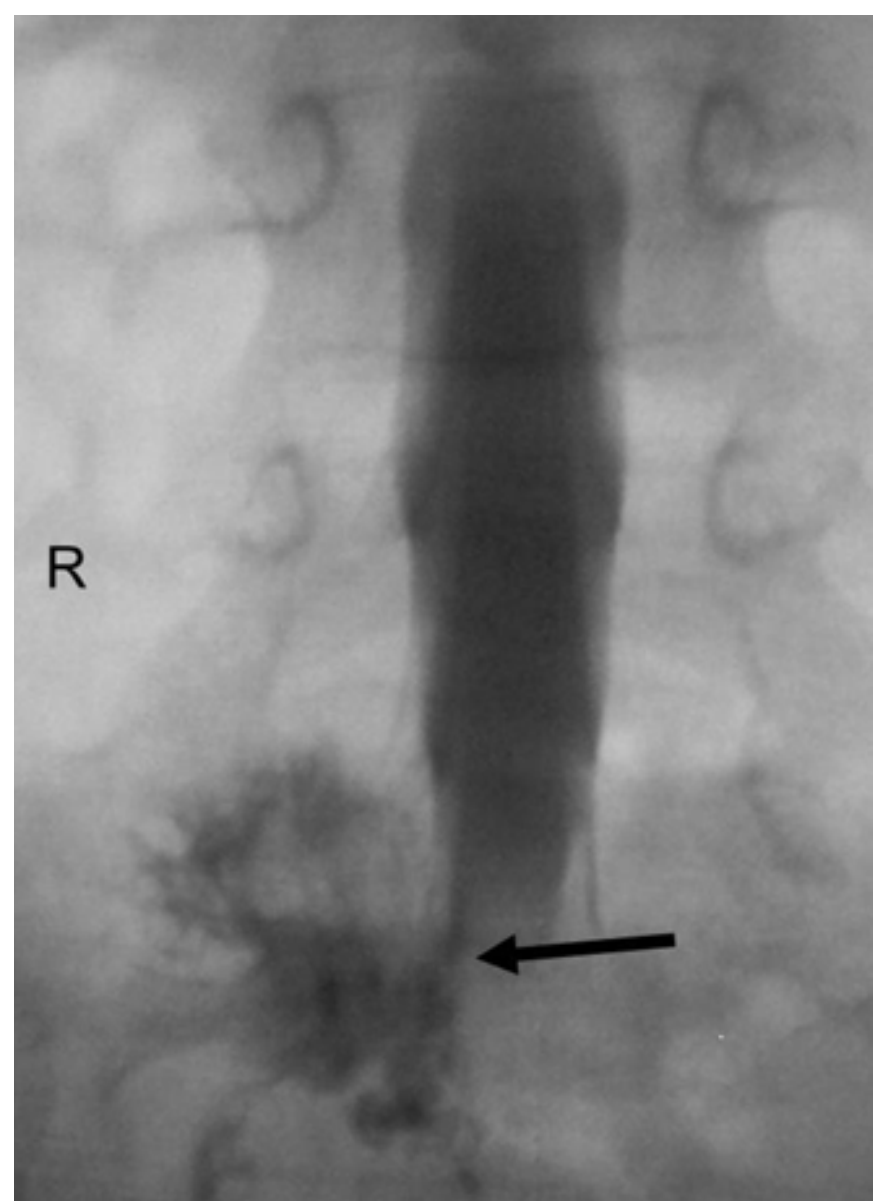

Figure 1: Myelogram done prior to first surgical repair. There is initial filling of the thecal sac with subsequent extravasation of the contrast from the right $S 2$ nerve root sleeve (arrow).

provide relief. Therefore, repair (of two small pin point dural defects leaking CSF around the axilla of the right S2 root found at surgical exploration ) was undertaken four weeks later.

Headaches resolved but recurred within a month, and began to occur daily; they were described as severe, with possible worsening in the upright posture. The MRI again demonstrated features diagnostic of SIH. Repeat CT myelography (Figure 2) demonstrated CSF leak around the right S3 nerve root (which may have been obscured by the more prominent leak from the right S2 nerve root sleeve in the earlier study). At surgery, the dura was attenuated around the axilla of the right S2 and S3 roots, and found to leak CSF. Muscle and blood patches were applied to these areas.

After a relatively symptom-free period of almost six months, headaches recurred and were apparently worsened during the upright position; MRI of the head showed features diagnostic for SIH (Figure 3). Computed tomogram myelography showed a reoccurrence of leak around the right S3 nerve root. At surgery, a small opening on the underside of the right S3 nerve root was found to be leaking CSF; it was sutured and a patch of muscle and fibrin sealant (Tisseel) placed over it.
The surgical findings suggested the possibility of developmentally weak meninges, and she was assessed in the Genetics Clinic. Clinical diagnostic criteria for Marfan and Ehlers-Danlos syndromes were not fulfilled. Cardiac and ocular assessments were normal. Full gene sequencing of the FBN1 gene did not identify any alterations.

Her headaches resolved gradually. There has been no clinical or radiological evidence of recurrence over the subsequent four years. She has developed mild kyphoscoliosis and is troubled by backache without any other clinical or radiological correlate.

\section{Discussion}

The diagnostic criteria for headache attributed to spontaneous (or idiopathic) low pressure (SIH; code 7.2.3 of ICHD-II), ${ }^{5}$ are shown in the Table. But, "it has become well established that the clinical and radiographic manifestations of SIH are highly variable," and in one study, only $3 \%$ of ninety subjects with neuroradiologically proven SIH satisfied ICHD-II criteria for the condition..$^{1,7-9}$ Postural headache although considered to be the clinical hallmark for SIH, (i) may not occur or do so long after being in the upright position, (ii) may not be relieved completely when supine, and (iii) if present, can disappear over time if SIH

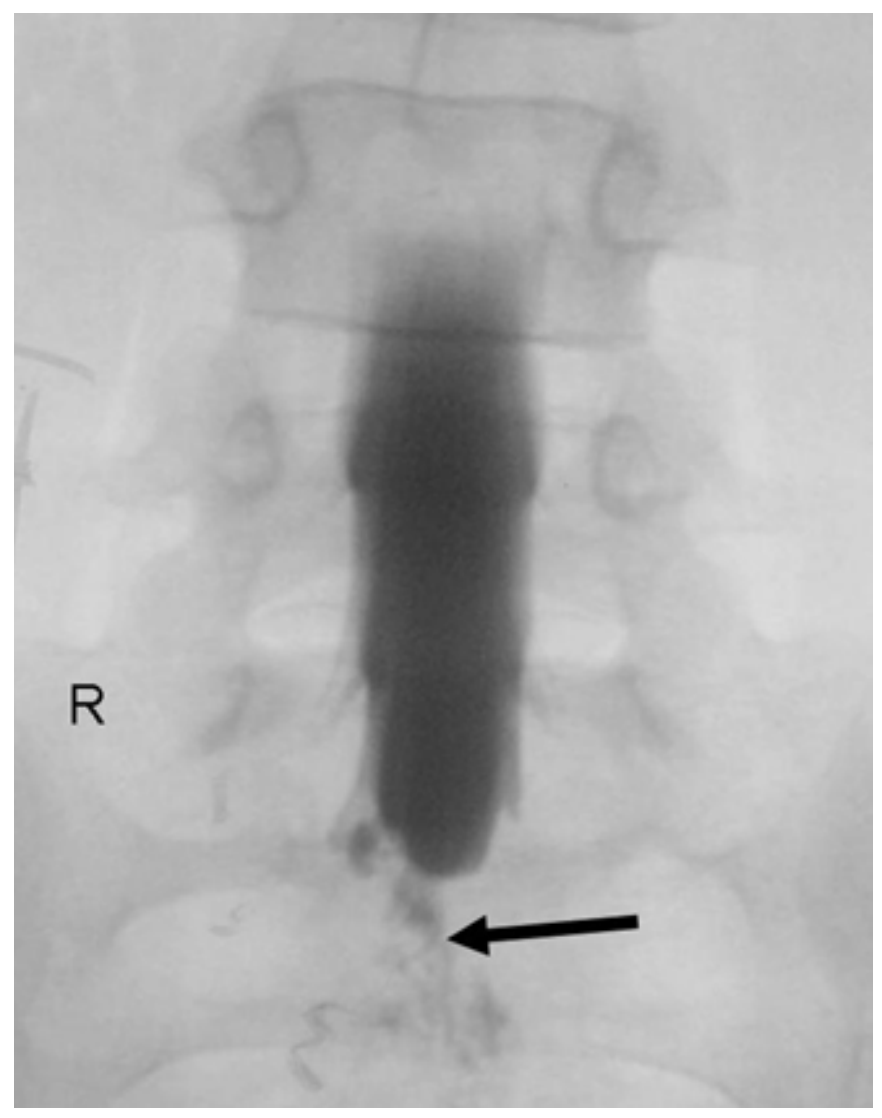

Figure 2: Myelography done a month after first surgical repair because of headache recurrence and MRI evidence of intracranial hypotension (see text). There is filling of the thecal sac with extravasation of contrast from the right $S 3$ nerve root sleeve (arrow). 


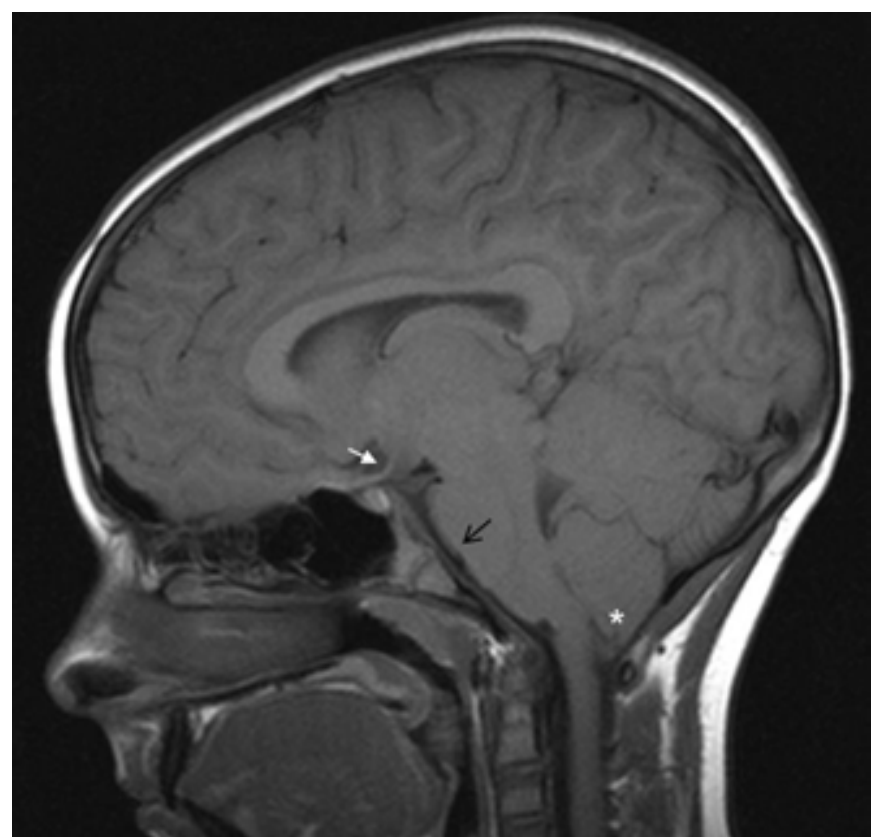

Figure 3: Unenhanced sagittal T1 weighted magnetic resonance image of the brain done six months after second surgical repair because of headache recurrence (see text). There is crowding of the posterior fossa structures with downward displacement of the cerebellar tonsils (*) below the level of the foramen magnum. There is "slumping" of the brainstem with flattening of the ventral pons along the clivus (black arrow). The optic chiasm is draped over the dorsum sellae (white arrow). is untreated. ${ }^{1,7,9}$ These authors also emphasized that orthostatic headaches were not always due to $\mathrm{SIH}$, and additional causes should "also be considered" in the differential diagnosis. Other headache patterns including nonorthostatic $\mathrm{CDH}$ (as in our case), paradoxical orthostatic headache (occurring when supine), and exertional headache have also been described in SIH; although location can be variable, occipital headache is common. ${ }^{1,9}$ The diagnosis in young children can be even more challenging, because of their limited developmental ability to describe symptoms. ${ }^{10}$

Spontaneous intracranial hypotension "nearly always results from CSF leaks."9 Mokri suggested that SIH due to spontaneous CSF leaks presented in four ways: (a) classically, with typical orthostatic headaches, low CSF pressures, and diagnostic neuroradiological findings all present, (b) with consistently normal CSF pressures, despite typical clinical and imaging findings, (c) without meningeal enhancement, despite typical clinical features and low CSF pressures, and (d) without headache, despite low CSF pressures and diagnostic radiological findings. ${ }^{9}$ In clinical practice, distinctions between normal and abnormal are rarely absolute, and uncertainty is common, as initially in our case.

Other clinical features in SIH include diplopia, ptosis, ataxia, facial numbness, back pain, stupor, coma and subdural hematoma. ${ }^{1,9,11}$ In adults, the presence of diplopia and absence of limb numbness had high sensitivity but low specificity in predicting CSF leaks in those who had a history of trauma; in those without a history of trauma, orthostatic headache was the

\section{Table: ICHD-II diagnostic criteria for Headache attributed to spontaneous (or idiopathic) low CSF pressure (SIH; Code 7.2.3)}

A. Diffuse and/or dull headache that worsens within 15 minutes after sitting or standing, with at least one of the following and fulfilling criterion D:
1. Neck stiffness
2. Tinnitus
3. Hypacusia
4. Photophobia
5. Nausea

B. At least one of the following:

1. Evidence of low CSF pressure on MRI (example, pachymeningeal enhancement)

2. Evidence of CSF leakage on conventional myelography, CT myelography or cisternography

3. $\mathrm{CSF}$ opening pressure $<60 \mathrm{~mm} \mathrm{H}_{2} \mathrm{O}$ in sitting position

C. No history of dural puncture or other cause of CSF fistula headache

D. Headache resolves within 72 hours after epidural blood patching

ICHD-II diagnostic criteria for Headache attributed to spontaneous (or idiopathic) low CSF pressure (SIH; Code 7.2.3). ICHD-II: International Classification of Headache Disorders, 2nd edition (Reference Number 5). CSF: cerebrospinal fluid; CT: computed tomographic; MRI: magnetic resonance imaging; SIH: spontaneous intracranial hypotension. Please note that the diagnostic criteria for CSF fistula headache (Code 7.2.2) are almost identical. Reproduced with permission of International Headache Society. 
only variable associated with CSF leaks; the authors drew attention to selection biases in their study, and suggested that their results might not be useful to screen for CSF leaks in the "general population". ${ }^{12}$ Trivial trauma around the time of symptom onset has been reported in a minority of patients; $;^{1,9}$ such history may be confounded by recall bias. The reporting of orthostatic headache during relapses after the first surgery in our case may have several explanations, including bias created by knowledge of the diagnosis.

The LP opening pressure has to be $<60 \mathrm{~mm}$ of water in the sitting position for diagnosis, ${ }^{1,5}$ but pressures may be normal or variable even in the same patient, ${ }^{9}$ as it was at the first LP in our case. Mokri suggested that depletion of CSF volume rather than low pressure was responsible for clinical features and neuroradiological findings. ${ }^{9}$ Continuous CSF pressure monitoring through the lumbar route can provide diagnostic information in selected cases of $\mathrm{CDH}$ without papilledema in whom idiopathic intracranial hypertension is suspected..$^{13}$ The procedure may have a similar role in subjects in whom SIH is strongly suspected but neuroradiological investigations and opening pressure at LP are normal. As in our case, the CSF may show increased number of red blood cells and leucocytes, and the CSF protein content may also be increased. ${ }^{1,9}$

Children with Marfan and related syndromes may be at risk for intracranial hypotension secondary to dural ectasia or arachnoid diverticulae, but most subjects with SIH only have isolated stigmata of heritable connective tissue diseases. . $^{1,3,9,14}$ Patients with SIH and their families should be assessed for the protean manifestations of an underlying inherited connective tissue disorder, recognizing that the clinical features may be isolated. Conversely, intracranial hypotension should be considered among the causes for headache in children who have features or family history of inherited connective tissue disorders, neurofibromatosis type 1, and polycystic kidney disease.

The relative diagnostic roles for CT scan of the head, MRI of the head and spine (with and without gadolinium), radionuclide cisternography, CT myelography, and MR myelography (with and without intrathecal gadolinium) have been discussed recently. ${ }^{1,15,16}$ Studies may be normal or findings subtle, as occurred initially in our case. There may be multiple sites of leak. ${ }^{1,16}$ Radionuclide cisternography can be useful in localizing the site of CSF leakage and guide detailed imaging with CT myelography.

Computed tomogram myelography is considered the investigation of choice for detecting CSF leaks. ${ }^{1,15}$ In children and adolescents, MRI myelography without intrathecal contrast (highly T2-weighted, fast spin-echo, fat-saturated sequences with thin sections) should be the initial procedure of choice to avoid radiation exposure. Several investigators have discussed the use of MR myelography with intrathecal gadolinium as an alternative to CT myelography, citing absence of ionizing radiation and greater sensitivity as advantages.,15 However, gadolinium is not approved for intrathecal administration in Canada or the United States. Invasive investigations should be avoided unless considered necessary for diagnosis and management.

Less than a third of cases with CSF leaks respond to epidural blood patching. ${ }^{1,7,9}$ Surgical treatment is not the focus of this paper, but "is not always straightforward," because the leaks are often multiple, and the anatomy complex, unlike "simple leaks that may occur after LP or epidural catheterization."9 The management of our case is in keeping with that suggested by Mokri and Schievink. ${ }^{1,9}$ Long-term data are scarce; rebound intracranial hypertension is a possible complication of surgical treatment, and relapse of SIH occurs in about ten percent. ${ }^{1}$

Despite advances in imaging techniques, the considerable variability in the clinical presentation, CSF findings and imaging abnormalities of SIH highlighted by Mokri, ${ }^{9}$ will continue to test our clinical abilities. The diagnosis of SIH can be overlooked by rigid adherence to ICHD-II criteria (Code 7.2.3). ${ }^{5}$ Therefore, more current guidelines are urgently needed to assist clinicians diagnose, investigate and treat $\mathrm{SIH}$ in children and adults. The possibility of SIH should be considered in any child who presents with $\mathrm{CDH}$, especially if headaches are predominantly occipital.

\section{ACKNOWLEDGEMENTS}

The radionuclide study was performed and interpreted by Dr. E.Duzic. The authors thank Dr. M. Seshia for reviewing the manuscript and the Faculty of Medicine and Department of Pediatrics, University of Saskatchewan for continuing support.

\section{Note}

References 1, 7, 9 and 15 are highly recommended for detailed reading. Standardized data sheets, discussed in other publications (SSS), were used to document information in the headache clinic. 


\section{REFERENCES}

1. Schievink WI. Spontaneous spinal cerebrospinal fluid leaks. Cephalalgia. 2008 Dec;28(12):1345-56.

2. Mokri B, Maher CO, Sencakova D. Spontaneous CSF leaks: Underlying disorder of connective tissue. Neurology. 2002 Mar 12:58(5):814-6.

3. Pyeritz R, Fishman E, Bernhardt BA, Siegelman SS. Dural ectasia is a common feature of the marfan syndrome. Am J Hum Genet. 1988;43:726-38.

4. Chan EK, Yan B, Ryan MM. Spontaneous intracranial hypotension in childhood: A case report and review of the literature. J Child Neurol. 2011 Jun;26(6):761-6.

5. Headache classification subcommittee of the International Headache Society. The International Classification of Headache Disorders. 2nd ed. Cephalalgia. 2004;24 (suppl 1):1-152.

6. Headache Classification Committee, Olesen J, Bousser MG, Diener $\mathrm{HC}$, et al. New appendix criteria open for a broader concept of chronic migraine. Cephalalgia. 2006 Jun;26(6):742-6.

7. Schievink WI, Dodick DW, Mokri B, Silberstein S, Bousser MG, Goadsby PJ. Diagnostic criteria for headache due to spontaneous intracranial hypotension: A perspective. Headache. 2011 Oct; 51 (9): $1442-4$

8. Mea E, Chiapparini L, Savoiardo M, et al. Application of IHS criteria to headache attributed to spontaneous intracranial hypotension in a large population. Cephalalgia. 2009 Apr;29(4): $418-22$.
9. Mokri B. Low cerebrospinal fluid pressure syndromes. Neurol Clin. $2004 \mathrm{Feb} ; 22(1): 55,74$, vi.

10. Andrasik F, Powers SW, McGrath PJ. Methodological considerations in research with special populations: Children and adolescents. Headache. 2005 May;45(5):520-5.

11. Chaudhary N, Cooper P, Lownie SP, Ng W, Duggal N. Spontaneous intracranial hypotension: Case series of rare clinical presentations. Can J Neurol Sci. 2011 Jan;38(1):54-8.

12. Ohwaki K, Yano E, Ishii T, Takanashi S, Nakagomi T. Symptom predictors of cerebrospinal fluid leaks. Can J Neurol Sci. 2008 Sep;35(4):452-7.

13. Torbey MT, Geocadin RG, Razumovsky AY, Rigamonti D, Williams MA. Utility of CSF pressure monitoring to identify idiopathic intracranial hypertension without papilledema in patients with chronic daily headache. Cephalalgia. 2004 Jun;24 (6):495-502.

14. Liu FC, Fuh JL, Wang YF, Wang SJ. Connective tissue disorders in patients with spontaneous intracranial hypotension. Cephalalgia. 2011 Apr;31(6):691-5.

15. Yuh EL, Dillon WP. Intracranial hypotension and intracranial hypertension. Neuroimaging Clin N Am. 2010 Nov;20(4): 597-617.

16. Wang YF, Lirng JF, Fuh JL, Hseu SS, Wang SJ. Heavily T2weighted MR myelography vs CT myelography in spontaneous intracranial hypotension. Neurology. 2009 Dec 1;73(22):1892-8. 